\title{
Labyrinthe
}

3 | 1999

Numéro 3

\section{La scène de genre dans les Salons de Diderot}

\section{Laurence Marie}

\section{(2) OpenEdition}

Journals

Édition électronique

URL : http://journals.openedition.org/labyrinthe/64

DOI : $10.4000 /$ labyrinthe.64

ISSN : 1950-6031

Éditeur

Hermann

Édition imprimée

Date de publication : 15 avril 1999

Pagination : 79-98

\section{Référence électronique}

Laurence Marie, «La scène de genre dans les Salons de Diderot », Labyrinthe [En ligne], 3 | 1999, mis en ligne le 07 février 2005, consulté le 23 avril 2019. URL : http://journals.openedition.org/labyrinthe/64 ; DOI : 10.4000/labyrinthe.64

Ce document a été généré automatiquement le 23 avril 2019

Propriété intellectuelle 


\title{
La scène de genre dans les Salons de Diderot
}

\author{
Laurence Marie
}

1 De 1759 à 1781, Diderot, répondant aux sollicitations pressantes de son ami Grimm, offre aux lecteurs de la Correspondance littéraire un compte rendu des Salons de l'Académie Royale de Peinture et de Sculpture, véritable institution qui prend place dans le Salon Carré du Louvre à un rythme bisannuel. L'objectif de la politique artistique qui s'exprime au Salon de l'Académie est de démontrer la suprématie de l'art français ad majorem regis gloriam et sa doctrine est celle de la hiérarchie des genres. Celle-ci est formulée clairement par Félibien dans sa Préface aux Conférences de l'Académie (1667) : en haut de l'échelle, on trouve la peinture d'histoire qui représente des thèmes de l'histoire religieuse et antique ou encore des sujets mythologiques ; puis, dans l'ordre, la scène de genre, le portrait, le paysage, et enfin la nature morte.

2 C'est seulement au XVIII siècle que se généralise le terme de " genre » pour désigner toute peinture qui n'est pas d'histoire, non sans paradoxe : ce terme définit d'abord le peintre qui n'est capable d'exceller que dans un seul genre et ne possède pas la capacité universelle souhaitée pour le peintre d'histoire. Le terme de genre désigne ensuite le peintre qui représente des scènes et des choses familières, appartenant à la vie courante, toujours par opposition avec le peintre d'histoire. Cependant, à l'intérieur de la peinture de genre, la scène de genre, scène d'intérieur, de mœurs, tient le haut du pavé, car elle représente un sujet animé. La représentation de cette réalité animée connaît ainsi une évolution fondamentale à la charnière des XVII et XVIII siècles. Les scènes de genre à la flamande, comme celles de Teniers ou de Brueghel mettent parfois l'accent sur les aspects marginaux ou caricaturaux de la vie quotidienne : rixes de mendiants, beuveries et autres " gueuseries ${ }^{1}$ » destinées à provoquer le rire du spectateur à travers la satire. Les scènes de genre flamandes ne se réduisent pourtant pas au comique, loin de là : bon nombre d'entre elles manifestent déjà une certaine volonté de distinction dans la représentation des personnages du commun. À partir de la fin du XVII ${ }^{e}$ siècle, la mise en scène traditionnellement comique d'une réalité considérée comme vulgaire tend désormais à se 
tourner vers le registre sérieux. C'est précisément de ce passage crucial du comique au sérieux que nous tenterons ici de rendre compte à la lumière des commentaires de tableaux offerts par les Salons de Diderot $^{2}$ : celui-ci doit en effet donner à voir les tableaux des Salons à des lecteurs absents. Les comptes rendus constituent ainsi le reflet des réflexions ambivalentes de l'époque sur le passage du comique au sérieux dans la scène de genre ; ils offrent également une description prolixe de scènes de genre pathétiques à la Greuze, autre voie de réhabilitation de la scène de genre. Pour parler à l'imagination du lecteur, l'écrivain doit lui-même participer à l'acte créateur et refait alors le tableau selon son goût : ce processus est révélateur de la conception de l'homme qui anime Diderot et ses contemporains.

3 La peinture de genre est tout d'abord définie par l'« idéal misérable ", par lequel Diderot caractérise Chardin. Elle est donc placée au bas de l'échelle dans la hiérarchie :

On a relégué dans la classe des peintres de genre les artistes qui s'en tiennent à l'imitation de la nature subalterne et aux scènes champêtres, bourgeoises et domestiques, et il n'y a que les peintres d'histoire qui composent l'autre classe qui puissent prétendre aux places de professeurs et à d'autres fonctions honorifiques ${ }^{3}$.

L'article « GENRE (peinture) » écrit par Watelet dans l'Encyclopédie, sous couvert de " donner aux gens du monde une idée plus noble qu'ils ne l'ont ordinairement des artistes qu'on appelle peintres de genre ", assied plus fortement encore la hiérarchie des genres. La peinture de genre reste pour lui destinée exclusivement aux peintres dont le talent est trop limité pour pouvoir s'élever à la grandeur de la peinture d'histoire : Watelet met en relation directe la médiocrité du talent et la restriction dans le choix des sujets, réels donc plus faciles à représenter.

5 La nature commune représentée par la peinture de genre - personnages de petit bourgeois ou de paysan - est donc jugée inférieure à la nature idéale de la peinture d'histoire: personnages historiques ou mythologiques. Diderot dit du Bénédicité de Chardin - qui met en scène une mère et ses deux enfants à l'heure du repas - qu'il traite d'une « nature basse, commune et domestique ». Le sujet bas s'oppose au sujet historique au même titre que l'anecdote à l'événement. Ainsi Diderot écrit :

Les peintres de genre et les peintres d'histoire n'avouent pas nettement le mépris qu'ils se portent réciproquement ; mais on le devine. [...] c'est la querelle de la prose et de la poésie, de l'histoire et du poème épique, de la tragédie héroïque et de la tragédie bourgeoise ${ }^{4}$.

6 Dans ses Réflexions critiques sur la poésie et la peinture (1719), Du Bos montre en effet pourquoi la bassesse de la scène de genre " ne saurait nous toucher ", à travers une esthétique qui privilégie l'expression des passions :

Il n'est rien dans l'action d'une fête de village ou dans les divertissements ordinaires d'un corps de garde qui puisse nous émouvoir. Il s'ensuit donc que l'imitation de ces objets peut bien nous amuser durant quelques moments, qu'elle peut bien nous faire applaudir aux talents que l'ouvrier avait pour l'imitation, mais qu'elle ne saurait nous toucher. Nous louons l'art du peintre à bien imiter, mais nous le blâmons d'avoir choisi pour l'objet de son travail des sujets qui nous intéressent si peu. [...] comment la copie me toucherait-elle si l'original n'est pas capable de me toucher ${ }^{5}$ ?

7 Plus loin, il affirme en outre que "l'homme qui passe son chemin », ou la " femme qui porte des fleurs » ne sont pas « intéressants », « intéressant » étant ici entendu au sens de « qui intéresse le sentiment ». 
8 Pourtant, la scène de genre, à l'intérieur de la peinture de genre, se situe en haut de la hiérarchie grâce à son caractère humain et donc animé. Elle est ainsi jugée supérieure à la nature morte par exemple. Dès 1668, alors que l'appellation peinture de genre n'existe pas encore, Félibien affirme le principe de la hiérarchie naturelle des genres fondée à la fois sur une hiérarchie naturelle de l'inanimé à l'humain, et sur la difficulté de la tâche de l'artiste. De plus, l'invention en 1712 du vocable «Fête galante » pour caractériser la peinture de Watteau souligne la reconnaissance officielle du droit de la peinture aux sujets dépourvus d'héroïsme. Si elle est relative, l'infériorité de la scène de genre reste cependant officiellement établie. Comment, malgré tout, racheter le " dégoût de l'objet » représenté ? Dans quelle mesure assiste-t-on au passage du comique au sérieux dans la scène de genre?

Il y a des sujets ingrats, mais c'est pour l'artiste ordinaire qu'ils sont communs. [...] Ah! Si un sacrifice, si une bataille, un triomphe, une scène publique pouvaient être rendues avec la même vérité dans tous ses détails qu'une scène domestique de Greuze ou de Chardin ${ }^{6}$ !

9 Cette exclamation où enthousiasme et regret sont mêlés souligne la principale qualité de la scène de genre, que la peinture d'histoire ne peut que lui envier : la " vérité dans tous ses détails ». Commentant l'allégorie de Lagrenée, L'Amour rémouleur, Diderot écrit : « Cela est infiniment moins vrai, moins intéressant, moins en mouvement que la même scène, si elle se passait dans la boutique d'un coutelier7. » Et d'ajouter : « Les peintres se jettent dans cette mythologie, ils perdent le goût des événements naturels de la vie ». La scène de genre mériterait-elle alors d'être à un rang supérieur à l'allégorie dans la hiérarchie des genres ? Tout au moins, seule la vérité de l'imitation permettrait à la scène de genre d'échapper à son caractère initialement comique. Diderot exhorte ainsi les peintres à se rendre dans la rue afin d'observer la nature « chez elle » et non pas « chez son voisin ${ }^{8} »$ :

Demain, allez à la guinguette ; et vous verrez l'action vraie de l'homme en colère.

Cherchez les scènes publiques ; soyez observateurs dans les rues, dans les jardins,

dans les marchés, dans les maisons, et vous y prendrez des idées justes du vrai mouvement dans les actions de la vie ${ }^{9}$.

10 L'artiste doit donc s'attacher à la vraisemblance de la scène représentée, pour que soit possible, ou du moins approchée, une imitation de la nature. Il lui faut rendre avec vérité « l'action ", par opposition à " l'attitude », l'action étant le miroir de l'intériorité des personnages. À propos du tableau de Roslin Un Père arrivant dans sa terre où il est reçu par ses enfants dont il était tendrement aimé, Diderot reproche avec indignation au peintre l'absence de vie et de vérité des membres de la famille représentée. Il se plaît ensuite à rapporter une proposition de Greuze destinée à faire vivre cette scène " plate et triste » : rassembler cette famille dans un salon le matin et laisser les hommes, les femmes et les enfants s'adonner à leur occupation favorite. L'expression des personnages, critère essentiel de lisibilité du tableau, doit elle aussi être vraisemblable : " L'expression est faible ou fausse si elle laisse incertain sur le sentiment ${ }^{10}$ ».

11 Lorsque les personnages sont expressifs quant à leur état et à l'action qu'ils effectuent, la description se constitue en véritable hypotypose ${ }^{11}$. À propos des Sevreuses, esquisse de Greuze $^{12}$, Diderot note : «Vous croyez être dans une chaumière, rien ne détrompe, ni la chose, ni l'art »; et va même jusqu'à inviter le spectateur à s'introduire avec lui dans le tableau, l'illusion étant alors presque totale :

Entrons dans cette cuisine ; mais laissons d'abord monter ou descendre cette servante qui nous tourne le dos, et faisons place à ce bambin qui la suit avec peine [...] Du pas de cette porte, je vois $[. . .]^{13}$ 
12 Parfois, le spectateur Diderot devient acteur à l'intérieur du tableau regardé, et l'espace de la toile prend alors toutes les apparences d'un lieu réel :

Je me trouve bien là, je resterai appuyé contre cet arbre, entre ce vieillard et sa jeune fille, tant que le jeune garçon jouera. Quand il aura cessé de jouer et que le vieillard remettra ses doigts sur sa balalaye, j'irai m'asseoir à côté du jeune garçon ${ }^{14}$

Les personnages picturaux s'animent et entament un dialogue avec le spectateur. Diderot s'adresse soudain en ces termes à La Jeune fille qui pleure son oiseau mort : « Pourquoi me fermer la bouche de votre main ? [...] vous souriez »; laquelle lui répond : « Et si la mort de cet oiseau n'était que le présage... Que ferais-je ? Que deviendrais-je ? S'il était ingrat ${ }^{15}$ $? »$

Comme le souligne le commentaire de La Jeune fille qui pleure son oiseau mort, les peintres contemporains de Diderot ont une prédilection marquée pour les personnages en état d'absorption intense. Starobinski le souligne : «ce qui parle dans la figure absorbée est le mystère d'une subjectivité qui demande à être interprétée, tout en refusant de se laisser deviner ${ }^{16}$ ». Cette ambivalence caractérise les personnages de Chardin en particulier ; c'est pourquoi ses toiles suscitent une émotion tour à tour sereine et inquiète. En effet, ces tableaux expriment un sentiment ténu, voire un refus de toute expression du sentiment ; cela confère, non sans paradoxe, un caractère éminemment mystérieux à ses toiles qui représentent pourtant des scènes parfaitement connues du spectateur : une mère et son enfant par exemple. "L'éloquence muette des actions quotidiennes et des objets communs ", leur " sereine monumentalité ${ }^{17}$ " font planer une menace de silence sur le discours de Diderot portant sur ces toiles. Paradoxalement, Diderot n'a pas suivi le chemin des ses contemporains qui mettent en avant la « distinction » des personnages de Chardin, pourtant issus du commun, et rapprochent ce peintre du genre de l'histoire au nom du contenu psychologique de ses scènes familiales. Les références à Chardin sont en effet assez rares dans les Salons : on trouve seulement une allusion au Bénédicité ${ }^{18}$, un bref commentaire de la Pourvoyeuse ${ }^{19}$ et une référence directe à L'Écureuse à propos de Bachelier. Cette dernière, la plus développée, exprime une forte émotion :

Il me semble que quand on prend le pinceau, il faudrait avoir quelque idée forte, ingénieuse, délicate ou piquante et se proposer quelque effet, quelque impression. Donner une lettre à porter est une action si commune qu'il faut absolument la relever par quelque circonstance particulière ou par une exécution supérieure. Il y a bien peu d'artistes qui aient des idées, et il n'y en a presque pas un seul qui puisse s'en passer. Oui sans doute, il est permis à Chardin de montrer une cuisine avec une servante penchée sur son tonneau et rinçant sa vaisselle, mais il faut voir comme l'action de cette servante est vraie, comme son buste dessine le haut de sa figure, et comme les plis de ce cotillon dessinent tout ce qui est en-dessous; il faut voir la vérité étonnante de tous les ustensiles de ménage et la couleur et l'harmonie de la petite composition ${ }^{20}$.

Le corps de la servante apparaît discrètement désirable et l'habituelle grivoiserie avec laquelle Diderot a coutume de traiter certaines figures féminines est ici absente. Le caractère suggestif des termes «buste » et " plis du cotillon » souligne cette sensualité voilée qui affleure. Celle-ci suscite une mystérieuse émotion, « aux limites de l'énonçable ", à cause de la basse condition de cette servante. Diderot semble dénier que ce corps puisse faire quelque effet sur lui autrement que par la peinture ${ }^{21}$. Néanmoins, cette allusion à L'Écureuse constitue un cas limite dans l'appréhension par Diderot de la scène de genre. Ainsi, à travers l'imitation fidèle de la nature animée, la scène de genre comique devient sérieuse et va même jusqu'à susciter une mystérieuse émotion, comme c'est le cas 
pour certaines toiles de Chardin. Cependant, si une copie fidèle de la nature n'est pas sans mérite, elle reste cantonnée à un rôle secondaire : ainsi, selon Diderot, si l'imitation de la nature ne rend jamais l'art maniéré, elle peut le rendre " pauvre, petit, mesquin ». « La nature commune fut le premier modèle de l'art. Le succès de l'imitation d'une nature moins commune fit sentir l'avantage du choix ${ }^{22} »$. Il ne s'agit pas de copier, mais de choisir la nature la plus forte, ce qui vaut la peine d'être peint. Ainsi est réaffirmée la supériorité de la peinture d'histoire, qui représente une nature idéale. C'est donc en empruntant à la peinture d'histoire certaines de ses caractéristiques - narrativité, expression des passions au service du pathétique - que la scène de genre peut trouver son rachat. Elle sera alors jugée comme la peinture d'histoire, à l'aune du sentiment produit sur le spectateur.

Revenons à la question de Du Bos : « Comment serions-nous touchés par la copie d'un original incapable de nous affecter? ». Il faut des sujets « intéressants », c'est-à-dire " qui intéressent le sentiment » : l'intensité des passions exprimées est jugée proportionnelle à l'effet produit sur le spectateur. Dans une esthétique qui privilégie l'expression des passions, ce n'est pas à travers la simple imitation de la nature animée que la scène de genre peut sortir de son infamie originelle, mais plutôt en mettant l'exactitude du genre au service d'une visée pathétique. La scène de genre emprunte à la peinture d'histoire sa grammaire du pathétique et la « réinjecte » dans une nature basse et commune.

Le peintre n'a qu'un instant, et il ne lui est pas plus permis d'embrasser deux

instants que deux actions ${ }^{23}$.

17 La peinture d'histoire et la peinture mythologique mettent en jeu l'intertextualité car elles s'appuient sur des textes antérieurs : elles font donc appel à la culture du spectateur qui peut reconstituer l'histoire racontée par le tableau. En revanche, la scène de genre est avant tout anecdotique car elle met en scène des personnages tirés du quotidien. Le peintre en est donc réduit à représenter des actions dans lesquelles le spectateur peut se reconnaître et imaginer l'avant et l'après de la scène : un moment de la vie quotidienne, comme le repas - Le Bénédicité de Chardin - ou un micro-événement de la vie familiale comme les fiançailles dans L'Accordée de village de Greuze. La mise en valeur de l'aspect narratif du tableau, procédé issu de la peinture d'histoire, permet ainsi de pallier la suspension du sens inhérente à la scène de genre. On assiste alors à une véritable théâtralisation du tableau.

18 Le peintre doit savoir choisir le meilleur moment et « relever une action commune par une circonstance particulière ». Diderot offre un commentaire narrativisé de La Jeune fille qui envoie un baiser par la fenêtre ${ }^{24}$ de Greuze, à travers l'emploi du présent et du passé composé à valeur d'accompli dans la description. Le temps est comme suspendu, et tout vient rappeler l'instant précédent : «Elle vient de recevoir un billet de son amant. Cet amant passe sous sa fenêtre et elle lui jette un baiser en passant [...] Le billet s'est échappé de sa main.»

19 Les accessoires, " micro-univers narratifs ", sont " chargés d'inscrire une dimension temporelle dans l'espace représenté ${ }^{25}$ ». Dotés d'un pouvoir en vertu duquel ils " gardent actualisé le moment précédent ", ils permettent ainsi de créer un effet de suspens au service du pathétique. Diderot met l'accent sur l'importance des accessoires à plusieurs reprises. À travers son commentaire du Fils puni, il écrit : « Greuze médite ses accessoires 
aussi sérieusement que le fond de son sujet ». Dans cette toile, en effet, le livre, la fiole et la bassinoire renvoient au moment précédent, c'est-à-dire ici à l'agonie du père.

La composition des tableaux est, elle aussi, au service du narratif. Plusieurs des scènes de genre présentées aux Salons de l'Académie mettent ainsi en scène des épisodes connexes : celles de Greuze en particulier développent une sorte de feuilleton pictural dont il n'est guère de vrai précédent que dans les cycles satiriques de Hogarth ${ }^{26}$. Ainsi, le diptyque narratif Le Fils ingrat et Le Fils puni de Greuze se rapproche des étapes " action » et " sanction " d'une tragédie bourgeoise en deux actes ${ }^{27}$. En outre, des tableaux n'ayant apparemment aucun rapport entre eux peuvent être associés à travers la composition plus ou moins cyclique des tableaux de Greuze ; certains personnages semblent en effet réapparaître d'une toile à l'autre : « Tout cela signifie que c'est sa petite Blanchisseuse d'il y a quatre ans qui s'est mariée, et dont il se propose de suivre l'histoire ${ }^{28} »$. La signification chez Greuze apparaît donc comme réticulaire : l'œuvre est une structure close où les personnages, devenus types, sont prisonniers d'un destin.

21 Associée au narratif, l'expression des passions, autre procédé emprunté à la peinture d'histoire, met en valeur le pathétique. Pour être immédiatement intelligibles, les passions ne doivent donc laisser place à aucune interprétation ambiguë. Greuze, à travers toute une série de gestes et de grimaces use d'une véritable grammaire du pathétique. Dans Le Fils ingrat et Le Fils puni ${ }^{29}$, les gestes des personnages sont comme suspendus dans un mouvement très théâtral exprimant les violentes passions qui les animent : dans le second tableau du diptyque, « la fille aînée assise dans le vieux confessionnal de cuir, a le corps renversé en arrière, dans l'attitude du désespoir, une main portée à sa tempe, et l'autre élevée et tenant encore le crucifix qu'elle a fait baiser à son père ». Les expressions du visage sont également représentées de telle sorte qu'on ne peut se tromper sur la nature de la passion qui anime le personnage : le fils ingrat « a l'air violent, insolent et fougueux »; le père est « indigné » ; la mère " a l'air accablé, désolé » L'article "PASSION (Peinture $)^{30} »$ de l'Encyclopédie donne un exemple de l'influence directe des passions sur le visage :

Dans la tristesse, les deux coins de la bouche s'abaissent, la lèvre supérieure remonte, la paupière est abaissée à demi, la prunelle de l'œil est élevée et à moitié relâchée, de sorte que l'intervalle qui est entre la bouche et les yeux est plus grand qu'à l'ordinaire, et par conséquent, le visage parait allégé.

Cette volonté de lisibilité conduit à une véritable typification des passions ${ }^{31}:$ chez Greuze, le fils est ingrat, le père autoritaire, la mère soumise, la fiancée timide...

L'expression des passions dans la scène de genre conduit ainsi à une extrême dramatisation dans les comptes rendus de tableaux, objets d'un fort investissement affectif de la part de Diderot. Commentant La Jeune fille qui pleure son oiseau mort de Greuze, il « se surprend conversant avec cette enfant et la consolant » et interprète la tristesse de la jeune fille comme causée par la perte de sa virginité, symbolisée par l'oiseau mort. Rien dans le tableau ne permet pourtant d'émettre une telle conjecture. À plusieurs reprises, il donne des conseils aux peintres dans le sens de la dramatisation et refait les tableaux selon son idée. À propos de La fille qui reconnaît son enfant à Notre-Dame parmi les EnfantsTrouvés, ou la force du sang ${ }^{32}$, il reproche à Baudoin d'avoir " réduit un événement pathétique à un incident qu'on devine à peine ». Après avoir décrit le tableau tel qu'il est, Diderot le réécrit tel qu'il devrait être, en mettant l'accent sur l'aspect narratif au service du pathétique. Le présent de narration crée un suspens et actualise la scène, ce qui renforce l'aspect pathétique : 
Voici comme il fallait s'y prendre et comme Greuze s'y serait pris. Je suppose qu'un père et une mère s'en soient allés à Notre-Dame avec leur famille [...]. Ils arrivent au banc des Enfants-trouvés [...]. L'aînée reconnaît son enfant ; à l'instant emportée par la tendresse maternelle qui lui fait oublier la présence d'un père à qui sa faute avait été cachée, elle s'écrie, porte ses deux bras vers cet enfant [...], la pâleur

24 explicite. Diderot s'exclame à propos de Greuze : «Voici votre peintre et le mien; le premier qui se soit avisé de donner des mœurs à l'art et d'enchaîner des événements d'après lesquels il serait facile de faire un roman ${ }^{33}$.» Le critique La Font de Saint-Yenne confère une fonction de moralité directe à la peinture qui doit " élever l'âme au-dessus des sens ${ }^{34} »$. Il parle alors de la peinture d'histoire mais Diderot, à travers la « peinture morale » de Greuze, semble transposer cette vocation à la scène de genre. Ainsi affirme-til lui aussi que la peinture se doit d'être bene morata ${ }^{35}$. « Rendre la vertu aimable, le vice odieux, le ridicule saillant, voilà le projet de tout honnête homme qui prend la plume, le ciseau ou le pinceau ${ }^{36}$.» Il s'exclame à propos du Fils puni : « Quelle leçon pour les pères et les enfants!».

Le repoussoir est ici incarné en la personne de Boucher, considéré comme l'apôtre des mauvaises mœurs : "Cet homme ne prend le pinceau que pour me monter des tétons et des fesses. Je suis bien aise d'en voir, mais je ne veux pas qu'on me les montre ${ }^{37}$. " De même, Diderot reproche à Baudoin l'ambiguïté morale perverse de ses toiles, et se livre à un commentaire des plus ironiques du Confessionnal ${ }^{38}$ qui représente deux jeunes égrillards qui se ruent sur des jeunes filles en pleine confession. Les toiles de ce peintre représenteraient alors un véritable danger pour les mœurs de la nation en général et pour l'éducation des jeunes filles en particulier. Diderot, en père soucieux de l'éducation de ses enfants, tempête : «il n'approchera pas de ma fille, ni lui, ni ses compositions ${ }^{39}$ ». En revanche, il loue le bon goût des épisodes choisis par Greuze ainsi que la décence de ses personnages : « Le choix de ses sujets marque de la sensibilité et de bonnes mœurs ${ }^{40}$ ».

L'alliance du narratif et du pathétique au sein du même tableau permet la création d'un nouveau genre qui rencontre "l'exactitude du genre et le sérieux de l'histoire ${ }^{41}$ ». Ce nouveau genre semble subvertir la division entre peinture d'histoire et peinture de genre au service de la morale : "Je ne rougirai pas d'avouer que Les Fiançailles de Greuze m'intéressent plus que le Jugement de Pâris ${ }^{42}$ ». Dans la même perspective, il écrit plus tard dans les Essais sur la peinture :

Il me semble que la division de la peinture en peinture de genre et peinture d'histoire est sensée, mais je voudrais qu'on eût un peu plus consulté la nature des choses dans cette division. [...] je proteste que Le Père qui fait la lecture à sa famille, Le Fils ingrat et Les Fiançailles de Greuze [...] sont autant pour moi des tableaux d'histoire que Les Sept Sacrements de Poussin, La Famille de Darius de Le Brun ou La Suzanne de Vanloo.

Cette revalorisation de la scène de genre par une « grammaire » importée de la peinture d'histoire conduit ainsi à un déplacement dans la division binaire genre / histoire : il semble que la distinction animé / inanimé apparaisse plus pertinente pour Diderot. Diderot va-t-il renverser la hiérarchie des genres au bénéfice de Greuze ? Cela semblerait logique puisqu'il préfère les Fiançailles de Greuze au Jugement de Pâris. Il ne le fait pourtant pas. Nous en apprenons la raison en lisant le passage où il commente le Septime Sévère en 1769 : 
Greuze est sorti de son genre : imitateur scrupuleux de la nature, il n'a pas su s'élever à la sorte d'exagération qu'exige la peinture historique. Son Caracalla irait à merveille dans une scène champêtre et domestique ; ce serait dans un besoin le frère de ce grand garçon qui écoute debout ce Vieillard qui fait la lecture à ses enfants.

Ainsi les Académiciens ont-ils bien fait, selon Diderot, de recevoir Greuze comme peintre de genre et non comme peintre d'histoire : il s'est fourvoyé en voulant sortir de sa spécialité et a fait un mauvais tableau. La revalorisation de la scène de genre picturale par l'injection du pathétique de la peinture d'histoire semble donc au demeurant bien limitée... La dimension sociale et idéologique attachée à la scène de genre l'explique : cette dernière représente en effet des personnages tirés du réel et souvent issus $d u$ commun, et quoi de plus polémique en ce siècle des Lumières?

L'amorce de revalorisation de la scène de genre semble menacée, voire annihilée, par la volonté de « distinction » qui anime Diderot et nombre de ses contemporains. On assiste en effet au XVIII siècle à un attachement beaucoup plus marqué à la hiérarchie sociale. Attachement qui n'était pas présent chez le chrétien Félibien : la représentation d'un prince n'était pas plus noble pour lui que celle d'un paysan. En revanche, au XVIII ${ }^{\mathrm{e}}$ siècle, s'opère une laïcisation : une hiérarchie sociale se substitue à la hiérarchie voulue par Dieu.

Au premier abord, Diderot ne montre pas de mépris particulier à l'égard des personnages du commun qu'il décrit, si l'on excepte quelques allusions plus ou moins grivoises portant sur des figures féminines. Néanmoins, il semble que c'est justement parce que ces personnages du commun sont pour ainsi dire à leur place dans la scène de genre considérée comme inférieure, qu'ils sont appréhendés sans ironie ni mépris. Ainsi, pour ces gens du commun, le peintre devra s'inspirer de modèles réels, alors que pour la peinture d'histoire ou mythologique, un modèle autre qu'idéal ne saurait se concevoir : à propos de La Poésie et la Philosophie, Diderot distingue modèle idéal et domestique ${ }^{43}$. De même, un tableau mythologique ne saurait ressembler de près ou de loin à une scène de genre. La hiérarchie des genres semble donc recouper la hiérarchie des modèles ; Diderot souligne à plusieurs reprises cette différenciation, à propos des personnages des tableaux historiques ou mythologiques : "L'Angélique est une petite tripière ; ô le vilain $\operatorname{mot}^{44}$ ! ", ou encore :

L'Anacréon est un charretier ivre, tel qu'on en voit sortir vers les six heures du soir des tavernes du faubourg Saint Marceau. Votre Diogène ressemble à un gueux qui tend la main de bonne foi ${ }^{45}$.

mais aussi à propos des actions de ces personnages : « On dirait que M. le prévôt des marchands invite Minerve et la Paix à prendre du chocolat ${ }^{46}$.»

La crispation sur la lisibilité de la hiérarchie sociale se traduit alors par le refus de la coexistence des classes sociales « hautes « et « basses « dans la même scène. Chacun se doit de rester dans son monde : le mélange du luxe et de la misère choque les bienséances sur un tableau :

Je suis révolté de la bassesse de cette vieille, de ces bottes de paille [...], et de cette élégante et de cet élégant qui la caresse. C'est du Fontenelle brouillé à du Théocrite [...] il n'entend rien à la convenance ${ }^{47}$.

ou au sein d'un même personnage :

Ce ne sont pas des tuiles, des chevrons, des toiles d'araignée qui sont vils, c'est un mélange de luxe et de pauvreté. Un paysan en sabots [...] , mouillé, crotté, vêtu de 
toile [...] est bien ; un laquais avec sa livrée usée [...], son vêtement tâché, est dégoûtant ${ }^{48}$.

Les deux adjectifs « bien » et " dégoûtant » en antithèse mettent en évidence la forte connotation morale et sociale de ce jugement. Ainsi Diderot semble-t-il projeter sur le tableau sa crainte du mélange entre le peuple et les personnes de la «bonne société », ce qui se manifeste par un attachement extrême à la lisibilité du tableau. À propos de L'Accordée de village de Greuze ${ }^{49}$, il insiste sur le problème d'identification de la jeune fille située derrière le père de la jeune promise : est-elle la sœur de l'accordée ou une simple servante $^{50}$ ? Le souci de lisibilité traduit la peur que le tableau ne conjure pas suffisamment l'idée d'une possible illisibilité de la société, la confusion qui règne dans le réel à l'époque.

La volonté de Diderot de se distinguer sans contredit des classes qu'il juge inférieures se retrouve même dans sa conception du spectateur :

Toujours petits tableaux, petites idées, compositions frivoles, propres au boudoir d'une petite-maîtresse, à la petite-maison d'un petit-maître ; faites pour de petits abbés, de petits robins, de gros financiers ou autres personnages sans mœurs et d'un petit goût.

L'anaphore de l'adjectif épithète antéposé " petit » souligne le mépris latent qui rappelle l'accusation d'immoralité et de mauvais goût professée contre Boucher. En revanche, $L a$ Cuisine italienne de Robert est une scène de genre à laquelle la lumière en demi-teinte confère une grande poésie, poésie à laquelle Diderot est fort sensible :

Ce morceau n'est pas fait pour arrêter le commun des spectateurs ; il faut à l'œil vulgaire quelque chose de plus fort et de plus ressenti ; ceci n'arrête que l'homme sensible au vrai talent $[\ldots]^{51}$.

Peut-être Diderot, à travers sa volonté de se distinguer du commun des spectateurs, tente-t-il de se justifier maladroitement d'avoir été profondément touché par ce tableau pourtant dépourvu de pathétique et de moralisme. En effet, aimer à contempler une scène de genre peut être, toujours selon le même Diderot, synonyme de mauvais goût et vecteur de disqualification sociale. Cet attachement quasi obsessionnel à la convenance sociale est peut-être ce qui l'empêche d'appréhender les scènes de genre, notamment celles de Chardin, dans toute leur dimension, ainsi que d'élaborer une théorie sur l'art qui soit vraiment autre chose que le reflet amplifié d'une époque. Les commentaires des scènes de genre témoignent cependant avec acuité de l'évolution qui touche la représentation de la famille au XVIII ${ }^{e}$ siècle : celle-ci occupe en effet une place centrale dans les toiles exposées au Salon de l'Académie.

Philippe Ariès met l'accent sur l'évolution fondamentale qui touche la famille et sa représentation aux XVII ${ }^{\mathrm{e}}$ et XVIII ${ }^{\mathrm{e}}$ siècles :

La «famille au XVII siècle n'est [...] pas la famille moderne : elle s'en distingue par l'énorme masse de sociabilité qu'elle conserve. Elle est [...] un centre de relations sociales, la capitale d'une petite société complexe et hiérarchisée que commande le chef de famille. Au contraire, la famille moderne se retranche du monde, et oppose à la société le groupe solitaire des parents et enfants. Toute l'existence du groupe est dépensée pour la promotion des enfants : les enfants, plutôt que la famille ${ }^{52}$.

À travers les Salons de Diderot, deux visions différentes de la famille coexistent : la famille au sens large représentée de façon pathétique et moralisante chez Greuze et la famille plus restreinte et intimiste de Chardin, à laquelle s'oppose Diderot.

Greuze, et Diderot dans ses comptes rendus de tableaux, offrent une vision idyllique de la prolifération des enfants, ce qui semble quelque peu démentir le malthusianisme dont on 
a taxé très tôt la bourgeoisie. Le commentaire de La Mère bien aimée ${ }^{53}$ donne l'image d'un père fier de son abondante progéniture : " C'est un jeune et gros garçon qui se porte bien et à travers la satisfaction duquel on discerne la vanité d'avoir produit toute cette jolie marmaille.» La petite morale qu'en tire Diderot est significative : «Cela est excellent et pour le talent et pour les mœurs ; cela prêche la population, et peint très pathétiquement le bonheur et le prix inestimable de la paix domestique. ». Greuze et Diderot, tout comme Rousseau professent une idéologie de la famille appuyée sur le pathétique. C'est au nom de cette idéologie que la scène de genre romanesque et picturale pourra entrer en concurrence avec l'histoire, de la même façon que le drame bourgeois avec la tragédie. En effet, les scènes familiales restent " concevables", mais à condition qu'elles contribuent explicitement au renforcement des idéaux sociaux : à travers les figures paternelles frappées ou menacées par la mort, le groupe social s'éprouve en danger, ce qui contribue à renforcer les liens familiaux, à " confirmer la cohésion des groupes sociaux élémentaires ${ }^{54}$ ». Ainsi, Diderot nomme Greuze " peintre de famille et d'honnêtes gens » et c'est cette peinture qu'il souhaite que l'on nomme d'histoire. En 1769, Greuze demande à l'Académie " une reconnaissance a posteriori de cette espèce en lui présentant son Septime Sévère à Caracalla ». Son tableau est refusé mais « il avait bien œuvré pour que la famille devînt le lieu d'un nouvel héroïsme ${ }^{55}$ ».

41 En revanche, ce n'est pas dans cette perspective que Chardin s'approche du genre de la peinture d'histoire, mais plutôt « dans sa manière de questionner les rapports humains à un endroit dont le siècle est en train de découvrir qu'il est fondamental ${ }^{56} »$. Cette peinture sérieuse, attentive aux sujets proches du spectateur, correspond ainsi à une réflexion où ce n'est pas dans les moments historiques que résident les moments importants pour l'homme, mais plutôt dans l'échange privilégié entre une mère et son enfant par exemple. Le Bénédicité de Chardin le souligne en mettant en scène une mère entourée de ses deux enfants attablés pour la prière précédant le repas. Il est significatif que ce soit la seule peinture familiale de Chardin à laquelle Diderot ait fait allusion dans les Salons, de façon fort dépréciative d'ailleurs : il parle à son propos d'une "nature basse, commune et domestique ». Ce commentaire sans appel met l'accent sur tout ce qui sépare Chardin d'un Greuze ou d'un Diderot: ce dernier ne conçoit pas en effet de peinture familiale dépourvue de toute connotation pathétique et morale.

Ainsi, la tentative de réhabilitation de la scène de genre par le passage au sérieux et au pathétique, si elle est limitée par la dimension sociale, n'en est pas moins réelle. L'évolution de la représentation du réel animé au XIX est encore marquée par cette ambivalence : pour ses scènes de genre, Gustave Courbet prônant la fidélité au réel, choisit délibérément ses sujets dans le quotidien le plus contingent ou le plus « choquant " pour le public de l'époque (La Toilette de la morte) faisant de ses toiles autant d'occasions de railleries ou de scandales. Cependant, le modèle pictural a aussi une portée pratique pour Diderot, dans Jacques le fataliste et son maître notamment. La scène du moine et des filles de joie, «tableau en récit », est particulièrement significative :

Jacques, en déshabillant son maître, lui dit : Monsieur, aimez-vous les tableaux ? LE MAÎTRE. Oui, mais en récit ; car en couleur et sur la toile, quoique j'en juge aussi décidément qu'un amateur, je t'avouerai que je n'y entends rien du tout ; que je serais bien embarrassé de distinguer une école d'une autre ; qu'on me donnerait un Boucher pour un Rubens ou pour un Raphaël ; que je prendrais une mauvaise copie pour un sublime original ; que j'apprécierais mille écus une croûte de six francs ; et six francs un morceau de mille écus; et que je ne me suis jamais pourvu qu'au pont Notre-Dame, chez un certain Tremblin, qui était de mon temps la ressource de la misère ou du libertinage et la ruine du talent des jeunes élèves de Vanloo. JACQUES. 
Et comment cela ? LE MAÎTRE Qu'est-ce que cela te fait ? Raconte-moi ton tableau, et sois bref, car je tombe de sommeil. JACQUES. Placez-vous devant la fontaine des Innocents ou proche la porte Saint-Denis ; ce sont deux accessoires qui enrichiront la composition. LE MAÎTRE. M'y voilà. JACQUES. Voyez au milieu de la rue un fiacre, la soupente cassée et renversé sur le côté. LE MAÎTRE. Je le vois. JACQUES. Un moine et deux filles en sont sortis. Le moine s'enfuit à toutes jambes. Le cocher se hâte de descendre de son siège. Un caniche du fiacre s'est mis à la poursuite du moine, et l'a saisi par sa jaquette ; le moine fait tous ses efforts pour se débarrasser du chien. Une des filles, débraillée, la gorge découverte, se tient les côtés à force de rire. L'autre fille, qui s'est fait une bosse au front, est appuyée contre la portière, et se presse la tête à deux mains. Cependant la populace s'est attroupée, les polissons accourent et poussent des cris, les marchands et les marchandes ont bordé le seuil de leurs boutiques, et d'autres spectateurs sont à leurs fenêtres. LE MAÎTRE. Comment diable ! Jacques, ta composition est bien ordonnée, riche, plaisante, variée et pleine de mouvement. À notre retour à Paris, porte ce sujet à Fragonard ; et tu verras ce qu'il en saura faire. JACQUES. Après ce que vous m'avez confessé de vos lumières en peinture, je puis accepter votre éloge sans baisser les yeux. LE MAÎTRE. Je gage que c'est une des aventures de l'abbé Hudson ? JACQUES. Il est vrai 57.

Cette anecdote semble être arrivée à Diderot lui-même, comme le montre une lettre à Sophie Volland ${ }^{58}$ et l'écrivain a d'ailleurs proposé ce sujet à Baudoin au Salon de $1765^{59}$. La description se constitue ainsi explicitement en tableau, à travers l'emploi du verbe voir, de l'adverbe " voilà » pris au sens étymologique mais surtout du présent et du passé composé qui créent instantanéité et simultanéité ${ }^{60}$. Dans Jacques le fataliste, Diderot se livre ainsi sans retenue aux " délices de la scène de genre ", son " faire " triomphe aux dépens d'histoires tournées en dérision. "Cette folle déliaison qui semble inspirée de l'ordre plus ou moins hasardeux imposé aux toiles d'un Salon ${ }^{61}$ », illustre la " théorie des riens ", par laquelle Marivaux justifie l'autobiographie ébauchée d'un petit voleur de pommes:

Vous vous étonnez qu'un Rien produise un si grand effet ; et ne savez-vous pas, raisonneur, que le Rien est le motif de toutes les plus grandes catastrophes qui arrivent parmi les hommes ? [...] les fameuses inutilités qui occupent aujourd'hui les hommes, et qu'on regarde comme le sujet des plus dignes travaux de l'esprit, sont peut-être, à qui les regarde comme il faut, de grands riens plus méprisables, ou pour le moins plus dangereux, que les petits riens semblables à ceux qui font en ce moment courir à ma plume la prétentaine sur le papier ; mais quittons un rien pour revenir à un autrés.

\section{NOTES}

1.Les exemples de ce type abondent dans les Salons : Le Cabaret de Casanove (Salon de 1767, p. 665), L'Intérieur d'un cabaret de Leprince (Salon de 1771, p. 904).

2.La pagination adoptée ici pour les Salons sera celle de la collection « Bouquins », Robert Laffont, tome IV.

3.Diderot, Salon de 1769. 
4.Essais sur la peinture, in CEuvres, T. IV, p. 505, coll. « Bouquins », Robert Laffont, Paris, 1996.

5.Sixième section (c'est nous qui soulignons), septième édition, Slatkine reprints, 1967. 6.Essais sur la peinture, p. 502 (c'est nous qui soulignons).

7.Salon de 1767, p. 560.

8.Salon de 1767, p. 582 : «La nature ! La nature ! Quelle différence entre celui qui l'a vue chez elle, et celui qui l'a vue en visite chez son voisin ».

9. Essais sur la peinture: « Mes pensées bizarres sur le dessin », p. 471.

10.Essais sur la peinture, p. 486.

11. "L'hypotypose peint les choses d'une manière si vive et si énergique, qu'elle les met en quelque sorte sous les yeux, et fait d'un récit ou d'une description, une image, un tableau ou même une scène vivante ", Fontanier, p. 290, cité par le Gradus de Dupriez.

12.Salon de 1765, p. 393.

13.Salon de 1767 : Robert, Cuisine italienne, p. 711.

14.Ibid.

15.Greuze, La Jeune fille qui pleure son oiseau mort, Salon de 1765, p. 382.

16.Jean Starobinski, « Diderot dans l'espace des peintres », dans Diderot et l'art de Boucher à David : les Salons, 1759-1781 (exposition), Hôtel de la Monnaie, 5 oct. 1984-6 janv. 1985, Paris.

17.Gita May, « Diderot entre le texte et l'image », Colloque international Diderot, 4-11 juillet 1984, actes réunis et préparés par A.-M. Chouillet, éd. Aux Amateurs de Livres, Paris, 1985. 18.Salon de 1761.

19.Salon de 1769, p. 843.

20.Salon de 1765 : à propos de tableaux à l'huile de Bachelier, p. 341.

21.René Démoris, Chardin, La Chair et l'objet, Paris, Éditions Adam Biro, 1995, p. 156.

22.Pensées détachées, " Du goût ", éd. citée, p. 1014.

23. Essais sur la peinture, « Paragraphe sur la composition », p. 496.

24.Salon de 1767, p. 437.

25.Christina Vogel, Diderot : l'esthétique des salons, thèse soutenue à Zurich en 1990, P. Lang, 1993, III 2 d, « La fonction des accessoires », p. 57.

26.L'organisation des images placées par cycles calqués sur le système dramatique permet à Hogarth (peintre et graveur anglais né en 1697 et mort en 1764) d'exposer implacablement, en les développant dans le temps, l'enchaînement des actes et des conséquences : Le Mariage à la mode (1744), La Carrière du roué (1733-1735), Le Zèle et la Paresse...

27. Christina Vogel, op. cit., III. 2.

28.Salon de 1765, à propos de L'Enfant gâté, p. 384.

29.Salon de 1765, p. 390-391.

30.Écrit par le Chevalier de Jaucourt.

31.Voir la typologie des passions établies par Descartes dans Les Passions de l'âme (1644).

32.Salon de 1765, p. 374.

33.Salon de 1765, p. 379.

34.La Font de Saint Yenne, Sentiments sur quelques ouvrages de peinture, 1754, réimpression Slatkine Reprints, Genève, 1970.

35. "La peinture a cela de commun avec la poésie, et il semble qu'on ne s'en soit pas encore avisé, que toutes deux elles doivent être bene moratae : il faut qu'elles aient des mœurs ", Essais sur la peinture, p. 500.

36. Essais sur la peinture, p. 501.

37.Salon de 1765, p. 312. 
38.Ibid., p. 372.

39.Ibid., p. 371.

40.Salon de 1761, p. 235 : à propos de L'Accordée de village de Greuze.

41.Jean Starobinski, L'Invention de la liberté : 1700-1789, Genève, Skira, 1964.

42.Salon de 1765, p. 328 : à propos de Quatre tableaux de la Vierge de Lagrenée.

43.Lagrenée, La Poésie et la Philosophie, Salon de 1767, p. 568-569 : « Les deux figures [...] ont un caractère domestique et commun qui ne convient guère à des natures idéales, abstraites, symboliques, qui devraient être grandes, exagérées et d'un autre monde. » 44.Salon de 1765 : Boucher, Angélique et Médor, p. 311.

45.Salon de 1767 : Restout le Fils, Les Plaisirs d'Anacréon, p. 760.

46.Salon de 1767 : Hallé, Minerve conduisant la paix à l'Hôtel de ville, p. 536.

47.Salon de 1767 : Baudoin, La Chaumière, p. 673.

48.Salon de 1767 : Baudoin, Le Sentiment de l'amour et de la nature cédant pour un temps à la nécessité, p. 671.

49.Salon de 1761.

50.« Autre défaut. Cette sœur aînée, est-ce une sœur ou une servante ? Si c'est une servante, elle a tort d'être appuyée sur le dos de la chaise de son maître, et je ne sais pas pourquoi elle envie si violemment le sort de sa maîtresse. Si c'est un enfant de la maison, pourquoi cet air ignoble, pourquoi ce négligé ?»

51.Ibid.

52.Philippe Ariès, L'Enfant et la vie familiale sous l'Ancien régime, Paris, Seuil, 1973, p.

306-307.

53.Greuze, Salon de 1765, p. 388.

54.Jean Starobinski, « Diderot dans l'espace des peintres », op. cit., p. 42.

55.René Démoris, Chardin, la chair et l'objet, Paris, Adam Biro, 1991, p. 125.

56.Ibid., p. 123.

57.Éditions Folio, p. 232-233.

58.7 octobre 1760 .

59.p. 375.

60.Nous renvoyons le lecteur à l'article d'Isabelle Vissière : « Une originalité de Diderot : le " texte-image " ", Diderot, les Beaux arts et la musique.

61.René Démoris, « Ut poesis pictura? ", dans Dilemmes du roman, Stanford French and Italian Studies 65, Anma libri.

62. Euvres de jeunesse, coll. « Bibliothèque de la Pléiade », p. 562-563.

\section{RÉSUMÉS}

La représentation du réel animé a connu une évolution fondamentale au XVIII ${ }^{\mathrm{e}}$ siècle dont les Salons de Diderot témoignent avec acuité. La représentation traditionnellement comique du réel considéré comme vulgaire tend alors à devenir un genre plus sérieux. Ainsi les scènes de genre de type flamand dans le style d'un Brueghel ou d'un Teniers ont laissé place à des peintures intimes de Chardin en particulier. Si Diderot peine à faire état de l'émotion paradoxale qui émane de ces dernières, ses commentaires des scènes de genre pathétiques à la Greuze sont au contraire 
prolixes. L'injection du pathétique dans la scène de genre, procédé utilisé dans la peinture d'histoire, est en effet le reflet d'une rhétorique qui privilégie l'expression des passions dans une visée morale. La volonté de destruction sociale qui motive Diderot et ses contemporains, bien que limitant la réhabilitation de la scène de genre, correspond cependant à une certaine conception de l'homme et de la famille.

The representation of the genre scene in Diderot's Salons. - The representation of the animate real has encountered a fundamental evolution in the eighteenth century demonstrated with acuity by the French writer Denis Diderot in his Salons. The traditionally comic representation of a real considered as vulgar tends then to become a more serious genre. Thus, the Flemish-type genre scenes in the manner of a Bruegel or a Teniers gave place to intimate paintings by Chardin in particular. If Diderot toils to report the paradoxical emotion which emanates from those paintings, his comments on pathetic genre scenes à la Greuze are on the contrary prolix. The "injection » of the pathetic in the genre scene, a process used in history painting, is indeed the reflect of a rhetoric that privileges the expression of the passions in a moral design. The willingness for social destruction that motivates Diderot and his contemporaries, although limiting the rehabilitation of the genre scene, corresponds however to a certain conception of man and family.

\section{AUTEUR}

\section{LAURENCE MARIE}

Laurence MARIE, née en 1976, est titulaire d'une maîtrise de Lettres modernes (Paris III). Son article est extrait d'un mémoire dirigé par René Démoris et intitulé : La Scène de genre : représentation du réel animé dans la peinture et le roman au XVIII siècle. Elle travaille actuellement à une maîtrise de Littérature anglaise (Paris III) portant sur l'intertextualité dans Arcadia et The Invention of Love de Tom Stoppard. 